\title{
The formation of discs in clusters
}

\author{
Paul C. Clark ${ }^{1}$
}

${ }^{1}$ Institute for Theoretical Astrophysics, University of Heidelberg

We review the properties of the discs that form around 'sink particles' in smoothed particle hydrodynamics (SPH) simulations of cluster formation, similar to those of Bate et al. (2003) and Bonnell et al. (2004), and compare them to the observed properties of discs in nearby star-forming regions. Contrary to previous suggestions, discs can form and survive in such an environment, despite the chaotic effects of competitive accretion. We find the discs are typically massive, with ratios of disc mass to central object mass of around 0.1, or higher, being typical. Naturally, the evolution of these discs is dominated by gravitational torques, and the more massive examples exhibit strong $m=2$ spiral modes. We also find that they can continuously grow over a period of 100,000 years, provided the central object is a single sink particle and the local density of sink particles is low. Discs that form around sink particles in the very centres of clusters tend to be shorter lived, but a single star can lose and gain a disc several times during the main accretion phase. However due to the nature of the turbulence in the cluster, the disc orientation can change dramatically over this time period, since disc-sink systems can accrete from counter-rotating envelopes. Since the competitive accretion process brings in material from large distances, the associated angular momentum can be higher than one would expect for an isolated star formation model. As such, we find that the discs are typically several hundred of AUs in extent, with the largest keplerian structures having radii of $\sim 2000$ AU. 